\section{EMPIRIC VERSUS ENTRAINED CAVOTRICUSPID ISTHMUS LINE ABLATION IN CONGENITAL HEART DISEASE: A SINGLE CENTRE EXPERIENCE}

Vinit Sawhney*, Alex Mclellan, Dhanuka Perera, Deshveer Babra, Dionisio Izquierdo, Richard Schilling, Pier Lambiase, Martin Lowe, Vivienne Ezzat. Barts Heart Centre

\subsection{6/heartjnl-2017-311726.77}

Introduction Catheter ablation for supraventricular tachycardia (SVT) in patients with congenital heart disease (CHD) is an important therapeutic option. Complex atrial substrate in this cohort makes atrial flutter (AFl) a common occurrence. However, induction of sustained tachycardia at the time of ablation is not always possible. We hypothesised that performing an empiric Cavotricuspid Isthmus CTI line ablation in case of non-inducibility of arrhythmia will lead to good outcomes. Long-term outcomes of empiric versus entrained CTI ablation in CHD patients with right-sided supraventricular arrhythmias were examined.

Methods and results 51 right-sided atrial flutter ablations in 49 patients from 2007-2015 were included. Data was analysed retrospectively. Medical records were reviewed and patients were contacted for follow-up. All patients with left sided arrhythmias were excluded. The mean age of the whole cohort was 43 years and $50 \%$ of the patients were men. Underlying aetiology included ASD (33.3\%), VSD (19.6\%), Fallot's tetralogy (15.6\%), Ebstein's anomaly (3.9\%), Fontan surgery (10\%) and Mustard repair (17.6\%). There was no significant difference in the right atrial sizes between the two groups. AFl was entrained in 34 patients whereas it was noninducible/non-sustained in 17 patients. The latter had an empiric CTI line ablation. Of those in the entrained group, $3 \%$ had other atrial arrhythmias induced in addition to typical AFl. $51 \%$ of procedures were performed without a general anaesthetic. There were no procedural complications. There was no significant difference in the mean procedure and fluoroscopy times between the two groups (Empiric vs Entrain CTI; 176.5 vs 163.8 and 41.5 vs $30.5 \mathrm{~min}$ ). After a mean follow-up of 21 months, arrhythmia-free survival was $71 \%$ versus $85 \%$ (p-value 0.21 ) in the empiric and entrained CTI ablation groups.

Conclusion Long-term outcomes after empiric and entrained CTI line ablation for AFl in patients with $\mathrm{CHD}$ are comparable. This is a safe and effective treatment option. In the case of non-inducibility of atrial flutter, an empiric CTI line ablation should be performed in this cohort.

\section{LONG-TERM OUTCOMES OF SUPRAVENTRICULAR TACHYCARDIA ABLATION IN CONGENITAL HEART DISEASE: SINGLE CENTRE UK EXPERIENCE}

Vinit Sawhney*, Alex McLellan, Dhanuka Perera, Dionisio Izquierdo, Deshveer Babra, Richard Schilling, Pier Lambiase, Martin Lowe, Vivienne Ezzat. Barts Heart Centre

10.1136/heartjnl-2017-311726.78

Introduction Catheter ablation for supraventricular tachycardia (SVT) in patients with congenital heart disease (CHD) is an important therapeutic option, which is safe and effective. Ablation outcomes vary significantly with increasing complexity of cases and data is limited. We reviewed the safety and long-term efficacy of SVT ablation in CHD patients and examined the differences in outcomes of simple and complex cases in a leading UK centre. We also examined the predictors of arrhythmia recurrence post ablation.

Methods and Results Consecutive patients undergoing SVT ablation from 2007-2015 from an independent prospective CHD registry were included. SVTs included AFl (typical/incisional/atypical), AVNRT and AVRT. Medical records were reviewed and patients were contacted for follow-up. 131 ablation procedures in 110 patients were included. Patients were divided into three groups based on complexity of cases. Group A (Simple CHD) - ASD/VSD/Valve disease, Group B (CHD of moderate complexity) - Fallots/Ebstein/Other and Group C (CHD of great complexity) - Fontan/Mustard/Total Cavopulmonary Connexion. Manual mapping and ablation was carried out in all cases using 3D electroanatomic mapping systems. The mean age of the cohort was 42 years and 52\% of the patients were men. $41 \%$ of cases were in Group C. Nearly half the procedures were carried out under general anaesthetic (49\%) with mean procedure and fluoroscopy times of 4 and 1 hours respectively. Acute ablation success was 95\%, with failure to terminate arrhythmia in three, unsuccessful transbaffle puncture in one and post-procedure complications in three patients (retroperitoneal haematoma, tamponade, $\mathrm{CHB}$ ). Over a mean follow-up of 2 years, $78 \%$ patients remained arrhythmia free. The mean arrhythmia recurrence time was 14 months. Two deaths were noted. Arrhythmia-free survival between the groups was not statistically significant (A, B, C; 76.5, 76.6, 72.2\%; p 0.9, 0.6, 0.6). There was a trend towards longer procedure times with increasing case complexity however this did not reach significance. Presence of AF (entire cohort) and LA diameter (Group C) correlated with arrhythmia recurrence $(\mathrm{p}=0.06,0.05)$.

Conclusion SVT ablation in context of CHD is safe and effective irrespective of the complexity of underlying anomaly. However, late recurrence is not uncommon. Presence of AF and atrial size is likely to predict outcome. There is scope to improve long-term single procedure efficacy (especially in complex cases) by using new technologies.

\section{RETROGRADE AORTIC ACCESS OF THE PULMONARY VENOUS ATRIUM PROVIDES EQUIVALENT OUTCOMES TO RIGHT ATRIAL OR TRANSEPTAL ACCESS OF THE LEFT ATRIUM IN PATIENTS WITH CONGENITAL HEART DISEASE}

Vinit Sawhney*, Alex McLellan, Dhanuka Perera, Dionisio Izquierdo, Deshveer Babra, Richard Schilling, Pier Lambiase, Martin Lowe, Vivienne Ezzat. Barts Heart Centre

\subsection{6/heartjnl-2017-311726.79}

Introduction Patients with congenital heart disease may suffer atrial arrhythmia where the target chamber can either be reached easily from a direct or transeptal approach, or may have a relatively excluded atrium where a retrograde aortic approach to the pulmonary venous atrium is required. We hypothesised that ablation outcomes in both groups will be similar.

Methods Retrospective analyses of patients with congenital heart disease (CHD) undergoing ablation of atrial arrhythmia at Barts Heart Centre over one year. Patients were divided into three groups based on access to the chamber of interest; Group 1) right atrial arrhythmia accessed directly; Group 2) left atrial arrhythmia accessed through the interatrial septum (transeptal puncture or via patent ASD); Group 3) arrhythmia 